\title{
Detecting intrinsic movements through needle tips pinned at Zusanli ST-36 point - a new method for characterizing structures for acupuncture treatments
}

\begin{abstract}
This study aims to investigate the characteristics of the observed intrinsic movements of needle tips pinned at the Zusanli ST-36 acupuncture point. The specificity of acupuncture points is an important principle underlying the acupuncture treatments and is closely related to the characteristics of some structures attributable to their therapeutic effects. The movements of needle tips pinned at the acupuncture and control points in rats were video recorded, and the position data were analyzed by using the power spectrum and the autocorrelation function. The power spectra in the frequency domain showed main peaks due to oscillations transferred from heart and lung movements, and the spectra showed no distinctive differences between the ST-36 and the control points. The autocorrelation function in the time domain, on the other hand, had different times for the first zero point in the envelope of the oscillating signals: $0.26 \pm 0.20$ minutes for the ST-36 site and $0.80 \pm 0.61$ minutes for the control site. The time difference was $0.54 \pm 0.45$ minutes, which was statistically significant with a p-value $=0.002$ from the paired t-test. The results of this study imply that the intrinsic movements transferred to the needle tips pinned at the ST-36 site are more disturbed by some internal mechanical factors than those transferred to the needle tips at the control site are and that the modernized method developed in this study may be a new tool for characterizing structures for acupuncture treatments.
\end{abstract}

Keywords: intrinsic movement, autocorrelation function, acupuncture point, needle, rat
Volume 3 Issue 6 - 2016

\author{
Jungdae Kim, ${ }^{1,2}$ Richard Cha, ${ }^{1,3}$ Minsun Lee, ${ }^{4}$ \\ Kwang-Sup Soh' \\ 'Nano Primo Research Center, Seoul National University, Korea \\ 2Pharmacopuncture Medical Research Center, Korean \\ Pharmacopuncture Institute, Korea \\ ${ }^{3}$ College of Physical Education, University of Suwon, Korea \\ ${ }^{4}$ Graduate School of Integrative Medicine, Sun Moon University, \\ Korea
}

Correspondence: Richard Cha, College of Physical Education, University of Suwon, 17,Wauan-gil, Bongdam-eup, Hwaseong-si, Gyeonggi-do, Republic of Korea, Tel 82318889382,

Email wellspring2@naver.com

Received: March 15, 2016 | Published: June 07, 2016

\section{Introduction}

Acupuncture as a traditional therapeutic intervention is practiced worldwide to treat patients for postoperative and chemotherapy nausea, vomiting, and many other painful conditions. ${ }^{1}$ The research field related to acupuncture has drastically expanded and matured over the last decade, but many unsolved puzzles persist, and new intriguing paradoxes have been emerging during the course of active studies. ${ }^{2,3}$ Numerous studies have investigated mechanistic models for acupuncture effects, and these models mainly focus on the effects of acupuncture needle stimulation of connective tissues, muscles, and the nervous system.

Considerable efforts have been made to understand the scientific mechanisms of acupuncture treatment based on anatomy, histology and physiology. ${ }^{4}$ Focusing on the neurological aspect, some modern physiologists suggest the "neural hypothesis," which states that the clinical influence of acupuncture is transferred primarily through stimulation of sensory nerves which provide signals to the brain. The information is then processed, resulting in the clinical changes associated with the treatment..$^{5}$ Therefore, the peripheral and the central nervous systems can clearly be considered to be the most plausible basis for the structure of the meridians and the acupuncture points. However, additional research is necessary to map precisely, based on the nervous system, the meridians and the acupoints from the skin to the brain. ${ }^{6}$

Connective tissue planes have been also proposed as anatomical and physiological equivalents to acupuncture meridians. ${ }^{7}$ The anatomical relationship of meridians and acupuncture points to connectivity tissue planes may be relevant to acupuncture's mechanism of action, suggesting a potentially important role for interstitial connective tissue. During acupuncture, the needle manipulation transmits a mechanical signal to connective tissue cells via mechano-transduction and that may explain the local and the remote effects of acupuncture. ${ }^{8}$ Such mechanistic models with the nervous system and connective tissue planes can be combined in such a way that our understanding of the effects of the stimulation by acupuncture needles should increase. ${ }^{2}$

One of the more refined questions arising from the study of acupuncture mechanisms concerns the specificity of acupuncture sites, the so-called point specificity. ${ }^{9}$ Point specificity is an important principle that underlies acupuncture treatment. Although point specificity in acupuncture is still a controversial matter, evaluations of the responses to stimulations at multiple points on the body's surface have shown that point-specific actions are present in hemodynamic, functional magnetic resonance imaging, and neurophysiological studies. ${ }^{10-12}$ In addition, point specificity is closely related to characterizing some structures attributable to the acupuncture effects. The characteristics of the structures for the acupuncture points and the meridians still remain as extensive research subjects in experimental laboratories. Recently, many works have used modernized, state-ofthe-art tools to measure the electrical, magnetic, optical, and chemical properties of acupuncture points and meridians. ${ }^{13-17}$

The main motivation of this work is derived from the old works done by Bong-Han Kim (BH Kim) and his colleagues who reported that the anatomical structure for the acupuncture and meridian system had been found through his experimental studies in the 1960s. Despite its potential importance in the basic science of biology and medicine, his report had been ignored for a long time mainly because the details of the protocols that they used were not published and because 
contemporary scientists had difficulty producing the same results. Only recently have most of his works been reviewed and confirmed by the team led by Prof. Kwang-Sup Soh. ${ }^{18}$

BH Kim demonstrated that his newly-found system, called the Bong-Han system (the primo vascular system is the currently accepted term), is composed of nodes and vessels. ${ }^{19-21}$ As one of characteristic features of the system, BH Kim described a very unique motion of the node that could be observed through the movement of the needle tip pinned in it. He called this special motion the Kim Se-Uk's phenomenon, which was named for the first observer, SeUk Kim, and his colleagues. His description of the phenomenon was so short that we were unable to reproduce the same results in the research. ${ }^{22}$ However, the basic idea was that the acupuncture needles showed different motions when they were pinned at the nodes under the acupuncture points. Motivated by this idea, we designed a more modernized version of animal experiments to observe this special motion and to introduce this special motion as a novel method for characterizing the acupuncture and meridian system. Because the anesthetized rats showed very subtle movements of the needle tips, a video-recording system was prepared to monitor the movements of the tips, and a new analysis method with the autocorrelation function was designed for analyzing the data from imaging processes.

\section{Materials and methods}

\section{Animal preparation and pinning the needle at the acupuncture point}

Nine-week-old Sprague-Dawley male rats were obtained from DooYeol Laboratory Animal Company (Seoul, Korea). The animals were housed in a controlled constant temperature environment $\left(23^{\circ} \mathrm{C}\right)$ with $60 \%$ relative humidity. All animals were exposed to a 12 -hour light-dark cycle and allowed access to food and water. Procedures involving the animals and their care conformed to the institutional guidelines (Approval No. WJIACUC 20140807-03-07).

The rats were anesthetized by using intraperitonial injections of urethane $(1.5 \mathrm{~g} / \mathrm{kg})$ in the abdomen. Hairs on lateral side of the hind limbs centered on the Zusanli point (ST-36) were removed by using a hair clipper and a depilatory (thioglycolic acid 80\%, Niclean cream, Ildong Pharm., Seoul, Korea). After 30 minutes in a stabilized condition, the rats were put on the stage for the experiments. For the selection of the acupuncture points in a rat model, we adopted the transpositional system proposed. ${ }^{23}$ In order to locate the ST-36 point, we first located the ST35 point, which is at the depression lateral to the patella ligament. The ST-36 point is located at the proximal one-fifth point on the line from the ST35 point to the anterior side of the ankle crease. We determined the position of the ST-36 point in the rats, ${ }^{23}$ and we selected the control point on the lateral side $2 \mathrm{~mm}$ from the ST-36 point as shown in Figure 1A. We also prepared the needles for palm acupuncture; the injecting tip was $2 \mathrm{~mm}$, and the total length, including the part for handling, was $20 \mathrm{~mm}$ (Korea Sujichim Company, Seoul, Korea), as shown in Figure 1B. This type of needle is frequently used in Korean traditional acupuncture over the area of the hand. Figure $1 \mathrm{C}$ shows the top view of needle pinned at the ST-36 or the control points.

\section{Experimental setup for recording the needle movements}

The experimental setup was composed of a video camera (Dr. Camscope, Sometech Company, Seoul, Korea), a halogen light source $(100 \mathrm{~W})$ and a stage for the rat. For vibration isolation, all instruments were put on an optical table with an air compressor so that any external effect on the observation of the intrinsic movements of the rat's body was minimized. The moving images taken with the video camera were transferred to a laptop computer and saved as movie files with a spatial resolution of $640 \times 480$ pixels for further image analysis. Figure $1 \mathrm{C}$ shows an enlargement of the tip of the handling part of the needle pinned in the rat's body for the analysis on the kinetics. The initial position of the tip in the field of view was adjusted by moving the camera in such a way that during the 10-minute observations, the tip was located mostly at the center of the screen view and never outside the monitoring area. After two needles had been inserted, one at the ST-36 point and the other at the control point, separate recordings for each tip were made for 10 minutes.

\section{Image processing for tracing the tip of needles}

Figure $1 \mathrm{C}$ shows a still image taken from the movie file. The image was focused on the tip of the needle, which was $500 \mu \mathrm{m}$ in width. The original 24-bit color image was inverted to an 8-bit gray image and put in binary format with a proper threshold value (200 in our case). For eliminating the noise in the image, we adopted the erosion and the dilation subroutines from the Open CV library (http://www. opencv.org). All the image processing and numerical analyses were done by coding the programs in $\mathrm{C}++$ language. The tip of needle may be traced by using the series of binary images. After having fixing the magnitude from the camera, we could deduce the actual sizes of the movements from the actual size of the needle tip in and the spatial resolution of the image. In this case, 1 pixel corresponds to $10 \mu \mathrm{m}$. By tracing the position of the needle tip, we were able to save the data in a text file for both the $\mathrm{x}$ - and the $\mathrm{y}$-axis position values on the twodimensional screen for further analysis.

\section{Spectral analysis and autocorrelation functions}

For the spectral analysis, we first chose one real-time dataset from among the position values along the $\mathrm{x}$ - and the $\mathrm{y}$-axis. If we denote the time-series raw data as $f(t)$ the power spectrum can be calculated by using the fast Fourier transform. We also obtained the autocorrelation function from the data as follows:

$$
R(\tau)=\int \tilde{f}(t) \tilde{f}(t-\tau) d t
$$

where the filtered data $f(t)$ were calculated from $f(t)$ by using a simple baseline wandering removal algorithm. The power spectrum shows the frequency information that is included in a relatively-short temporal behavior of the data. Through the autocorrelation function, on the other hand, we were able to determine the long-term behaviors from the 10-minute data.

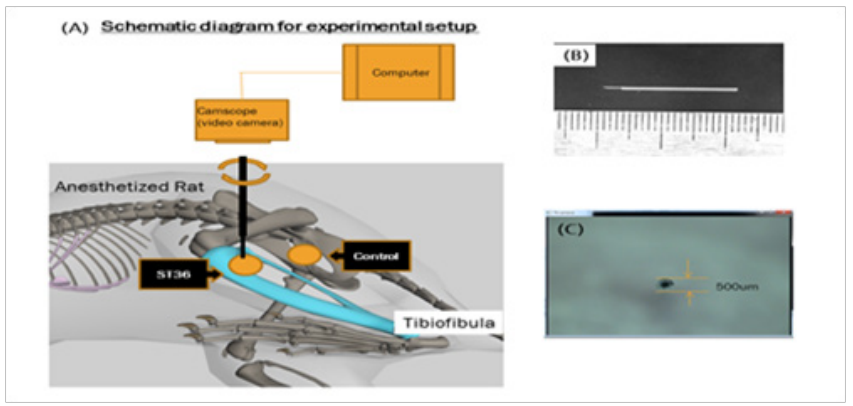

Figure I (A) Schematic diagram for experimental setup composed of a video recording system and an anesthetized rat on the stage and pinned with the needles. (B) The needle that is commonly used for hand acupuncture. (C) The needle tip for imaging and recording with the camscope. 


\section{Results and discussion}

Figure 2 shows typical data extracted from the recorded movie files on the motions of the needle tips after image processing. The movements of the tips pinned at the ST-36 point are shown in the combined plot of the (x,y) positions in Figure 2(left panel). Actually, the data from the ST-36 and the control points were taken by consecutive measurements on the tips pinned at the two sites. In this case, the scanned areas of the ST-36 and the control points for the 10-minute observations were within the ranges of $\Delta \mathrm{x} \sim 500 \mu \mathrm{m}$, $\Delta \mathrm{y} \sim 200 \mu \mathrm{m}$, and $\Delta \mathrm{x} \sim 200 \mu \mathrm{m}, \Delta \mathrm{y} \sim 100 \mu \mathrm{m}$, respectively. The realtime data for the movement of the tip along the $\mathrm{x}$-axis for 10 minutes are shown in Figure 2(right panels). We note that the directions of movements, $\mathrm{x}$ - or $\mathrm{y}$-axis, do not change the overall results from the analyses. The size of movements tends to decrease with time because of the stable conditions during the measurements.
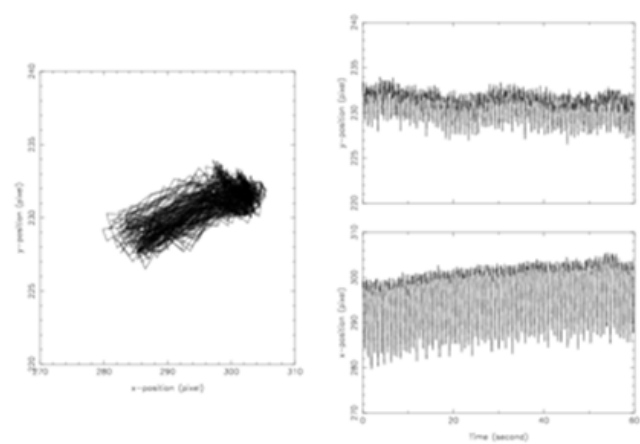

Figure 2 Movements of the needle tip: (left panel) a two-dimensional $x-y$ plot after tracing and saving the positions of the tips and (right panels) a onedimensional plot of the tip's movements along the $\mathrm{x}$-axis (the width direction of the screen) and the $y$-axis for 10 minutes.

The typical frequency properties of the real-time data can be extracted by using the power spectrum (Figure 3). The first peak in the spectrum at a frequency of $1.36 \mathrm{~Hz}$ is due to respiration. After the 2nd and 3rd harmonics of the respiratory frequency, another peak, which can be attributed to the beating of the heart, is seen at $4.7 \mathrm{~Hz}$. The primary frequencies from the motions of the heart and the lungs are followed by a series of peaks from the harmonics. The normal physiological parameters for the rats were as follows: a heart rate of $250 \sim 370$ beats/min and a respiration rate $70 \sim 115$ breaths $/ \mathrm{min}$. The frequency ranges for the motions of the heart and the lungs correspond to $4.17 \sim 6.17 \mathrm{~Hz}$ and $1.17 \sim 1.92 \mathrm{~Hz}$, respectively. ${ }^{24}$ The dominant peaks are mainly due to the motions of the heart and the lungs even though the measurements were carried out on the hind limb of the rat. The frequency patterns from the ST-36 and the control points in the power spectrum almost overlap, so identifying any characteristic feature with the spectral analysis was difficult. The other internal sources of motion may be gastric and bowel movements, for which the rates are about 5 counts per minute $(0.08 \mathrm{~Hz})$ in average rats. In our case, some peaks in the time series data may be interpreted as being a gastric slow wave, but generally those peaks were not well enough defined for further analysis.

The autocorrelation functions were calculated with the measured data along the $\mathrm{x}$-axis and the $\mathrm{y}$-axis from eight rats, and the typical results are shown in Figure 4. In some cases of rats, we measured the movements on both sides of the rats' legs. In the other cases, the left side was the focus of the measurements. In general, the autocorrelation functions decrease as time passes and re-emerge again in fluctuating patterns. At a certain time, the so-called 'first zero point,' the envelopes for the autocorrelation function have their first zero values (nodes) between fluctuating nodes (anti-nodes). Figure 4 shows the autocorrelation plots based on the $\mathrm{x}$-axis data for three rats. In the figure, the first zero points of the envelope are indicated by arrows. If the times of the first zero points from the data for the ST-36 and the control points are compared, almost all the times for the ST-36 point are shorter than those for the control point. In order to eliminate any individual differences among the rats, we also consider the time difference, and the data are shown in Table 1. The time difference between the ST-36 and the control points is statistically significant with $p=0.002$ from the paired t-test. Similar patterns are observed for the autocorrelation functions calculated with the y-axis data. The differences are just the amplitudes of the autocorrelation functions, and the positions of the first zero points are exactly the same as they are for the $\mathrm{x}$-axis data.

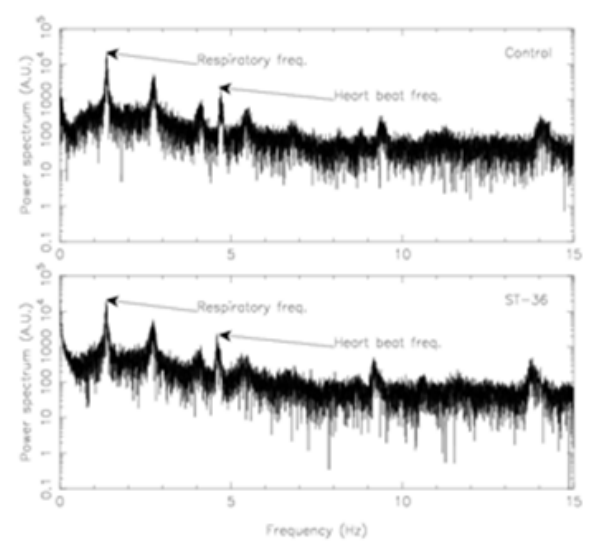

Figure 3 Power spectra calculated from the time series data on the movements of the tips pinned at the control site (top panel) and ST-36 (bottom panel).

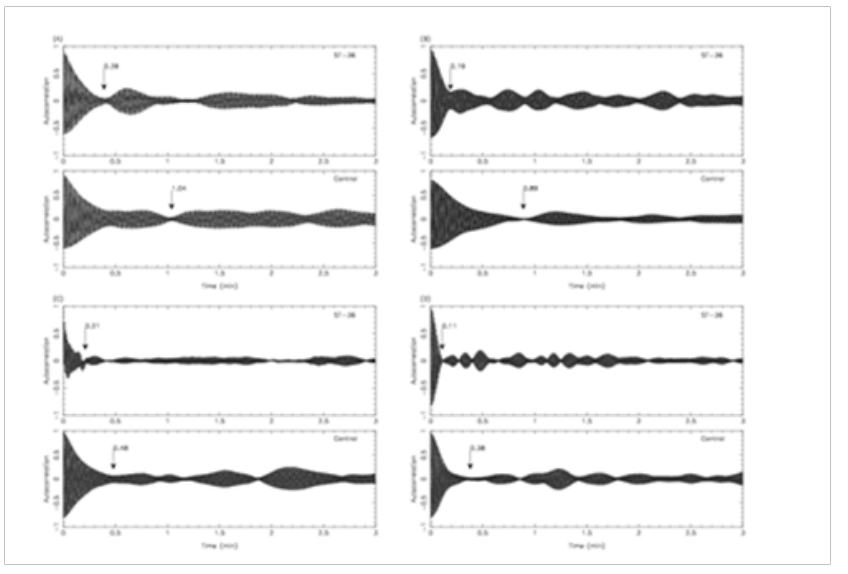

Figure 4 Typical autocorrelation functions calculated from the $x$-axis data. The arrows indicate the positions of the first zero in the envelope of the oscillating signals.

(A) Rat $\mathrm{I}$ in the left side, (B) Rat 2 in the left side, (C) Rat 3 in the left side, and (D) Rat 4 in the right side.

Table I The times in minutes for the first zero point in the autocorrelation functions measured at the ST-36 site and at the control site

\begin{tabular}{lllll}
\hline Rat no. & Side & ST-36 & Control & (Control)-(ST-36) \\
\hline Rat I & left & 0.39 & 1.04 & 0.65 \\
Rat 2 & left & 0.19 & 0.89 & 0.70 \\
\hline
\end{tabular}


Table Continued

\begin{tabular}{lllll}
\hline Rat no. & Side & ST-36 & Control & (Control)-(ST-36) \\
\hline Rat 3 & left & 0.21 & 0.48 & 0.27 \\
Rat 3 & right & 0.11 & 0.38 & 0.27 \\
Rat 4 & left & 0.28 & 0.48 & 0.20 \\
Rat 4 & right & 0.24 & 0.60 & 0.36 \\
Rat 5 & left & 0.22 & 0.31 & 0.09 \\
Rat 6 & left & 0.75 & 2.35 & 1.60 \\
Rat 7 & left & 0.05 & 0.39 & 0.34 \\
Rat 8 & right & 0.16 & 1.03 & 0.87 \\
Average $\pm S D$ & & $0.26 \pm 0.20$ & $0.80 \pm 0.61$ & $0.54 \pm 0.45$ \\
\hline
\end{tabular}

Autocorrelation is a cross-correlation of a time series signal with itself at different points in time and shows the similarities between observations as a function of the time lag between them. The autocorrelation function is one of many mathematical tools for finding repeating patterns, such as the presence of a periodic signal obscured by an unexpected perturbation and/or noise, or for identifying some missing fundamental frequency in a signal implied by its harmonic frequencies. Signal processing is frequently used to analyze a series of values such as time-domain signals. The power spectrum and the autocorrelation functions are related to each other by the Wiener-Khinchin theorem. ${ }^{25}$ Basically, both contain the same information because the same time series data are used for the calculations. However, their ways of presentations are completely different and complementary in a sense. While the power spectrum shows the frequency information in the frequency domain, the autocorrelation function shows the coherence information in the time domain. As shown in our results, contrary to the spectral analyses, the autocorrelation functions clearly show separable patterns in the movements of the ST-36 and the control points, which may imply that the intrinsic movements transferred to the needle tips pinned at the ST-36 site are more disturbed by some internal mechanical factors than the needle tips pinned at the control site are. These results did not seem to depend on the order in which the measurements at the two points and on the two sides of the rats were made.

Many studies have examined the feasibility of quantifying acupuncture needle manipulation by using motion and force measurements under the hypothesis that distinct needling styles and techniques would produce different force patterns and needle motions. $^{26}$ The motion patterns during acupuncture manipulation have been examined to evaluate the consistencies in acupuncture manipulation. ${ }^{27,28}$ In addition, an in-vitro study investigated collagen fiber alignment induced by acupuncture needle manipulations. ${ }^{29}$ Most of those studies focused on the extrinsic movements of the needles and the stimuli-response effects. In this study, we used a video-recording system with a magnified view of the small motions of the needle tips, which may be introduced as a new method for characterizing acupuncture points, to investigate the intrinsic movements.

As shown in Figure 3, several peaks in harmonics higher the 2nd harmonic originated from the periodic oscillations of the heart and the lungs. These unwanted peaks are usually due to some asymmetries in the oscillations and to the finite number of data points. Figure 4 shows that the autocorrelation functions have many zero points after the first zero point in time. In general, the amplitudes of the autocorrelation functions decrease with passing time. In some cases, the second zero point is off the time axis; thus, a longer measurement time is needed.

The limitations of our current work are as follows: First, because only the ST-36 acupoint was considered, a generalization of the results to other acupoints may not be possible. Second, the mechanical source for the internal factor to which the shortening of the first zero point of the autocorrelation functions may be attributed could not be established.
The first limitation can be overcome by conducting more systematic experiments including other acupoints. Simultaneous measurements with extended video resolution and better image processing algorithms may expand the observed areas around the acupoint sites. The second limitation needs more elaborate research to clarify the mechanical source. One possible solution may be the Bong-Han system, as the original idea of Se-Uk Kim's observation implies. The tip movements at the non-ST-36 points or their corresponding adjacent non-acupoints have been measured for comparisons with those at the ST-36 points in the experiments. The difference of the autocorrelation will be very crucial point that represents an anatomical difference between them and that induces different physiological responses. Our findings from the animal experiments can be applicable to humans for further clinical study on acupuncture mechanism.

\section{Conclusion}

In conclusion, the movements of needle tips pinned at the ST36 acupuncture point were video recorded, and position data were analyzed by using the power spectrum and the autocorrelation function. The most dominant motions came from the heart and lungs, which was confirmed by the frequency analysis. The autocorrelation functions showed clear distinctions between the motions at the ST-36 point and those at the corresponding control point, which implies that this method may be a new tool for characterizing the structure for acupuncture treatments.

\section{Acknowledgments}

None.

\section{Conflicts of interest}

Author declares there are no conflicts of interest.

\section{Funding}

None.

\section{References}

1. NIH. NIH Consensus Conference. Acupuncture. JAMA 1998;280(17):1518-1524.

2. Napadow V, Ahn A, Jonghurst J, et al. The status and future of acupuncture mechanism research. J Altern Complement Med. 2008;14(7):861-869.

3. Langevin HM, Wayne PM, MacPherson H, et al. Paradoxes in acupuncture research: Strategies for moving forward. Evid Based Complement Alternat Med. 2011;2011:180805.

4. Goldman N, Chen M, Fujita T, et al. Adenosine A1 receptors mediate local anti-nociceptive effects of acupuncture. Nat Neurosci. 2010;13(7):883-888.

5. Burnstock G. Acupuncture: a novel hypothesis for the involvement of purinergic signaling. Med Hypotheses. 2009;73(4):470-472.

6. Longhurst JC. Defining meridians: a modern basis of understanding. $J$ Acupunct Meridian Stud. 2010;3(2):67-74.

7. Langevin HM, Yandow JA. Relationship of acupuncture points and meridians to connective tissue planes. Anat Rec. 2002;269(6):257-265.

8. Langevin HM, Churchill DL, Cipolla MJ. Mechanical signaling through connective tissue: a mechanism for the therapeutic effect of acupuncture. FASEB J. 2001;15(12):2275-2282.

9. Campbell A. Point specificity of acupuncture in the light of recen clinical and imaging studies. Acupunct Med. 2006;24(3):118-122. 
10. Choi EM, Jiang F, Longhurst JC. Point specificity in acupuncture. Chin Med. 2010;7:4.

11. Ren Y, L Bai J, Tian J, et al. Investigation of acupoint specificity by functional connectivity analysis based on graphy theory. Neurosci Lett. 2010;482(2): 95-100.

12. Xing J, Zeng BY, Li J, et al. Acupuncture point specificity. Int Rev Neurobiol. 2013;111:49-65.

13. Lee MS, Jeong SY, Lee YH, et al. Differences in electrical conduction properties between meridians and non-meridians. Am J Chin Med 2005;33(5): 723-728.

14. Yang HQ, Xie SS, Liu SH, et al. Differences in optical transport properties between human meridian and non-meridian. Am J Chin Med. 2007;35(5):743-752.

15. Ahn AC and Martinsen OG. Electrical characterization of acupuncture points: technical issues and challenges. J Altern Complement Med. 2007;13(8):817-824.

16. Park SH, Kim J, Koo TH. Magneto-acupuncture stimuli effects on ultraweak photon emission from hands of healthy persons. $J$ Acupunct Meridian Stud. 2009;2(1):40-48.

17. Yan X, Zhang X, Liu C, et al. Do acupuncture points exist? Phys Med Biol. 2009;54(9):N143-150.

18. Soh KS. Bonghan circulatory system as an extension of acupuncture meridians. J Acupunct Meridian Stud. 2009;2(2):93-106.

19. Vodyanoy V, Pustovyy O, Globa L, et al. Primo-vascular system as presented by Bong Han Kim. Evid Based Complement Alternat Med. 2015;2015:361974
20. Stefanov M, Kim J. Primo vascular system as a new morphofunctional integrated system. J Acupunct Meridian Stud. 2012;5(5):193-200.

21. Kang KA. Historical observations on the half-century freeze in research between the Bonghan system and the primo vascular system. $J$ Acupunct Meridian Stud. 2013;6(6):285-292.

22. Kim BH. On the Kyungrak system [in Korean]. J Acad Med Sci DPRK. 1963;90:1-35.

23. Yin CS, Jeong HS, Park HJ, et al. A proposed transpositional acupoint system in a mouse and rat model. Res Vet Sci. 2008;84(2):159-165.

24. Davis JA. Mouse and rat anesthesia and analgesia. Curr Protoc Neurosci 4: Appendix 4B. 2008.

25. Chatfield C. The Analysis of Time Series-An Introduction. 4th edn London: Chapman and Hall; 1989. p.94-95.

26. Davis RT, Churchill DL, Badger GJ, et al. A new method for quantifying the needling component of acupuncture treatments. Acupunct Med. 2012;30(2):113-119.

27. Seo Y, Lee IS, Jung WM, et al. Motion patterns in acupuncture needle manipulation. Acupunct Med. 2014;32(5):394-399.

28. Lee IS, Lee YS, Park HJ, et al. Evaluation of phantom-based education system for acupuncture manipulation. PLoS One. 2015;10(2):e0117992.

29. Julias M, Edgar LT, Buettner HM, et al. An in vitro assay of collagen fiber alignment by acupuncture needle rotation. Biomed Eng Online. 2008;7:19. 